\title{
Atributos Preferidos por los Estudiantes de Ingeniería Comercial en la Enseñanza de la Asignatura Administración de Empresas
}

\author{
Sebastián C. Araya-Pizarro*, Carlos P. Varas-Madrid y Luperfina E. Rojas-Escobar \\ Universidad de La Serena, Facultad de Cs. Sociales y Económicas, Dpto. de Cs. Económicas y \\ Empresariales, Amunátegui s/n, La Serena-Chile. (e-mail: saraya@userena.cl; cvaras@userena.cl; \\ Irojas@userena.cl) \\ * Autor a quien debe ser dirigida la correspondencia
}

Recibido Abr. 28, 2017; Aceptado Jul. 5, 2017; Versión final Sep. 4, 2017, Publicado Feb. 2018

\begin{abstract}
Resumen
El presente estudio analiza los atributos preferidos en la enseñanza de la asignatura Administración de Empresas, materia basal de la carrera universitaria de mayor matrícula en Chile: Ingeniería Comercial. En base a la revisión bibliográfica, entrevistas y grupos de discusión, se seleccionaron cinco atributos relevantes: nivel de exigencia, tipo de clase, uso de tecnologías de información y comunicación, número de profesores y estilo docente. Estos atributos fueron examinados a través de la técnica multivariante de Análisis Conjunto. Los resultados muestran que, independientemente del género y edad, los estudiantes se inclinan por una clase con alto nivel de exigencia, que distribuye equilibradamente las horas de teoríapráctica, que emplea tecnología multimedia y que es dictada por un solo profesor con un estilo integrador de enseñanza. Se espera que las preferencias declaradas por el alumnado contribuyan a ajustar las prácticas educativas a objeto de mejorar su motivación y rendimiento académico.
\end{abstract}

\section{Attributes Preferred by Students of Commercial Engineering for the Teaching of the Subject Business Administration}

\begin{abstract}
The present study analyzes the preferred attributes for teaching of the Business Administration subject, a fundamental course of the career with the highest enrollment in Chile: Commercial Engineering. Based on a bibliographical review, interviews and focus group, five relevant attributes were selected: level of demand, class type, use of information and communication technologies, number of teachers and teaching style. These attributes were examined using the multivariate Conjoint Analysis technique. The results show that, independent of gender and age, students prefer a high-level requirement class, with an equal number of hours of theory and practice, which use multimedia technology and which is taught by a single teacher with an integrating teaching style. It is expected that the preferences declared by the students will contribute to adjusting the educational practices in order to improve their motivation and academic performance.
\end{abstract}




\section{INTRODUCCIÓN}

Los nuevos escenarios del siglo XXI demandan a las instituciones de educación superior, nuevos requerimientos y desafíos de formación profesional. La discusión sobre la calidad de las instituciones universitarias y de la educación que se imparte, ha motivado la revisión de los actuales procesos de enseñanza/aprendizaje y de las prácticas de evaluación, llevando a los planteles educativos a reorientar la configuración de sus planes estratégicos de desarrollo. Además, el fenómeno de la deserción universitaria continúa avanzando pese a los esfuerzos de las instituciones académicas y las autoridades por revertirlo. En Chile, por ejemplo, de acuerdo al Servicio de Información de Educación Superior (SIES) se calcula que el sistema de educación superior pierde, en promedio, un 30\% de los estudiantes que ingresan a primer año, identificando como causa principal el bajo rendimiento académico logrado que los desmotiva a continuar. Todo lo anterior, hace imperativo reconocer los factores que los estudiantes y docentes consideran relevantes y por tanto prefieren al momento de recibir e impartir una clase, entendiendo por ésta: la unidad funcional donde se tratan los contenidos, de una asignatura, en toda su dimensión a partir de la selección adecuada del método de enseñanza, que conlleva al cumplimiento de los objetivos propuestos y la transformación integral pronosticada en el alumno.

La carrera de Ingeniería Comercial es una rama de la Ingeniería Administrativa que busca desarrollar profesionales con competencias en gestión estratégica, operaciones, marketing y negocios. Su origen se remonta al año 1903 en la Ecole de Commerce Solvay, Universidad Libre de Bruselas. En Chile, sus inicios se sitúan en el año 1935, con la creación de la Escuela de Ingeniería Comercial de la Universidad de Chile (López y Paredes, 2007) y actualmente representa la carrera con mayor matrícula y empleabilidad de la nación. Entre los países que ofrecen esta carrera universitaria se encuentran: Argentina, Bolivia, Chile, Colombia, Ecuador, Guatemala, República Dominicana, México, Nicaragua, Panamá, Paraguay, Perú y San Marino. En la Región de Coquimbo (Chile), la carrera de Ingeniería Comercial se dicta en cinco universidades, siendo la Universidad de La Serena, el único plantel estatal que la imparte. El programa se adscribe a la Facultad de Ciencias Sociales y Económicas de la Universidad de La Serena y declara como objetivo formar profesionales con conocimientos, habilidades y actitudes necesarias para administrar negocios y empresas, bajo un Modelo Educativo centrado en el estudiante. De esta manera, la asignatura de Administración, constituye un pilar fundamental de su malla curricular, sobre todo si se considera que expone como una competencia clave dentro de su programa de estudios: "Administrar y gestionar empresas, negocios, y funciones organizacionales tanto en el sector público como privado".

La presente investigación definió como propósitos específicos: (i) identificar las dimensiones de una clase de administración, más valoradas por los alumnos de primer año de la carrera de Ingeniería Comercial de la Universidad de La Serena; (ii) evaluar la utilidad individual y agregada de los distintos niveles de cada atributo y (iii) determinar la contribución de cada uno de los atributos en las preferencias indicadas por los alumnos. Para la valoración de los atributos y la evaluación de la estructura de las preferencias se utilizó la técnica multivariante conocida como Análisis Conjunto. En particular se espera que los hallazgos encontrados puedan contribuir a mejorar la impartición de la asignatura en cursos académicos posteriores y, de esta manera, reducir los niveles de repitencia y deserción de las nuevas generaciones de estudiantes.

La teoría de la utilidad representa el mayor aporte teórico para examinar las preferencias por un bien o servicio. Comprende el concepto de utilidad como el grado en que un bien puede satisfacer la necesidad de un sujeto, a través de la apreciación subjetiva que éste efectúa sobre sus cualidades tangibles e intangibles. Para su estimación, se presentan el enfoque cardinal, destacado por economistas como Bentham, Senior, Dupuit, Jevons y Marshall, que supone que es posible cuantificarla, por medio de una unidad de medida de la satisfacción. Y el ordinal, impulsado por Hicks, Pareto, Kaldor y Samuelson, que señala que el consumidor sólo establece combinaciones de bienes que prefieren o que le son indiferentes por sobre otros. En ese contexto, para la medición de las preferencias se encuentran dos grandes vertientes. La primera, conocida como preferencias reveladas la que indica que éstas pueden determinarse basados en un principio de racionalidad fundado en el comportamiento observado del consumidor que después puede ser utilizado para abordar su función de utilidad (Nicholson y Snyder, 2008); y segundo, los métodos de preferencias declaradas, que se basan en la opinión de los individuos sobre la manera que actuarían frente a diferentes situaciones propuestas (experimentos), con las cuales se genera la información necesaria para calcular las funciones de utilidad vinculadas a cada una de las alternativas presentes en el experimento (Louviere et al., 2000). Dentro de las técnicas de las preferencias declaradas, resaltan los métodos de valoración multiatributo, y en especial, el modelo de preferencias del Análisis Conjunto.

El Análisis Conjunto es una de las principales técnicas de análisis multivariante, utilizada para el estudio de las preferencias de los individuos (Farías y Fistrovic, 2016; García-Vidal et al., 2016; Gómez-Valenzuela, 2015; Huertas-García et al., 2016). En ella, el investigador, describe productos o servicios a través de un conjunto de características (atributos) con la idea de medir el nivel de utilidad que el usuario le otorga, 
considerando, por lo general, que la preferencia de una alternativa es una función aditiva de las utilidades parciales (partworths) que la constituyen. De esta forma, los dos supuestos que subyacen en el análisis conjunto son: (i) el comportamiento de elección del consumidor o usuario se rige por la maximización de las preferencias, y (ii) un producto o servicio puede ser visto como un conjunto de atributos a partir de los cuales los usuarios obtienen su utilidad (Ramírez et. al, 2004). Dentro de las ventajas del análisis, se encuentra que entrega una visión más realista del problema decisional, al brindar al usuario la posibilidad de realizar comparaciones (tradeoffs) entre las opciones, además de que permite estimar en qué proporción influye cada atributo en sus preferencias (importancia relativa).

Dentro del ámbito de la investigación son numerosas las publicaciones que analizan los aspectos influyentes en la enseñanza (y aprendizaje) de una materia o un programa educativo. A continuación, se presenta una breve revisión bibliográfica respecto de los cinco atributos significativos para el trabajo. El estudio acerca del Nivel de exigencia de una clase ha sido abordado desde la perspectiva de las prácticas de evaluación, donde en lo referente a los requerimientos ideales de lo que se presume debiera ser un buen sistema curricular, los estudiantes esperan altas exigencias (Huaquín y Loaíza, 2004), con evaluaciones que presenten un nivel de dificultad alto, pero coherentes a la complejidad de las clases (Asun et al., 2013). Por su parte, en el análisis sobre el Tipo de clase, se constata la importancia de adoptar diferentes tipologías (teórica y práctica) para favorecer la formación integral de los alumnos (Borgobello et al., 2010; HernándezInfante e Infante-Miranda, 2017) y la necesidad de articularlas adecuadamente considerando las preferencias actuales de los estudiantes por un enfoque de instrucción práctico (Kharb et al., 2013; Ramírez et al., 2008). En el ámbito del Uso de las TIC existe consenso en señalar que las herramientas y técnicas de enseñanza deben ajustarse al contexto tecnológico actual de la sociedad del siglo XXI (Ajani, 2016; Ubulom et al., 2016), de modo que es posible encontrar diversos estudios comparativos que confirman la inclinación de los estudiantes por el uso de material multimedia en vez de los recursos educativos tradicionales (Boyer et al., 2009; Robinson y Stubberud, 2012; Tárraga et al., 2012).

En relación al Número de profesores en una clase, ésta tradicionalmente se ha efectuado de manera individual, pero existen casos que destacan experiencias colaborativas de dos o más docentes. La coenseñanza si bien posibilita a los profesionales que comparten instrucción, combinar sus conocimientos y habilidades para crear nuevos ambientes formativos (Suárez-Díaz, 2016), también presenta el desafío de llevarse bien, utilizar estilos de enseñanza, habilidades y áreas de conocimiento complementarios entre sí y preocuparse de diseñar, adecuadamente, la planificación de la clase (Ainscow, 2012; Ramos, 2016). Respecto al estudio de preferencias de los estudiantes sobre el número de profesores en una clase se ha encontrado el favoritismo por tener un solo docente dedicado a la teoría y práctica (Ramírez et al., 2004).

Por último, las investigaciones modernas referente a los Estilos de enseñanza docente destacan la tendencia por el uso de estilos de aprendizaje activos por sobre los tradicionales (Cui, 2013; GonzálezPeiteado, 2013; Rojas-Jara et al., 2016), vinculados a estilos de liderazgo docente más transformacionales, donde prima la motivación y estimulación de la capacidad analítica de los estudiantes para lograr sus objetivos en contraposición a estilos transaccionales, en que el profesor intercambia calificaciones y recompensas por el esfuerzo de los alumnos (Cerdá y Ramírez, 2010; Maureira y Rojas, 2013).

En un nivel multiatributo, Ramírez et al. (2004), analizaron cuáles son las características de la docencia de la asignatura de matemáticas que más valoran los alumnos de primer curso de la Licenciatura en Administración y Dirección de Empresas de la Universidad Pablo de Olavide. A través del Análisis Conjunto, concluyeron que, lo que más valoran los alumnos es la distribución de los tipos de clase (entre teoría y práctica), la duración de las clases y el número de profesores. Menores importancias concedieron al horario, al tamaño de los grupos y a las tutorías. Por otra parte, Kuzmanovic et al. (2013), aplicaron el Análisis Conjunto en el estudio sobre la evaluación de la enseñanza en la Universidad de Belgrado (Serbia). Luego de analizar nueve atributos de tipo cualitativo, demostraron que el criterio más importante fue una presentación clara y comprensible, seguidas de un enfoque metódico y sistemático, tiempo de la clase y disponibilidad de los docentes. Estos cuatro factores explicaron en casi un $60 \%$ el total de las preferencias de los encuestados.

\section{MATERIALES Y MÉTODOS}

En virtud de los objetivos planteados en la investigación, se consideró apropiado la utilización de un estudio descriptivo, transeccional con enfoque predominantemente cuantitativo. La investigación examinó, a través del cálculo de índices de utilidad marginal, los atributos de una clase de Administración más valorados por los estudiantes pertenecientes a la carrera de Ingeniería Comercial de la Universidad de La Serena (ULS). Para el proceso de medición se utilizó la técnica estadística de interdependencia conocida como Análisis Conjunto (Conjoint analysis) que permitirá caracterizar la función de utilidad de los sujetos determinando la importancia relativa que cada atributo tiene en la enseñanza de la materia. 
En un inicio, con el fin de conocer los atributos del proceso de aprendizaje más importantes para los estudiantes, se llevó a cabo una revisión bibliográfica de la literatura existente, junto con la aplicación de dos focus group (de 10 participantes cada uno) a alumnos de diferentes niveles de la carrera de Ingeniería Comercial de la ULS $\left(2^{\circ}, 4^{\circ}\right.$ y $5^{\circ}$ año); y siete entrevistas a expertos de la ULS, que incluyeron: 2 directores de Escuela, 3 académicos de la carrera, el Jefe de la Unidad de Mejoramiento Docente (UMD) y el tutor profesional del Centro de Apoyo al Aprendizaje. Como resultado, se obtuvieron, seis atributos y 12 niveles de valoración, los que fueron medidos, posteriormente, en una encuesta personal aplicada a una muestra estratificada de 72 estudiantes de pregrado de Ingeniería Comercial de la ULS, a través de un muestreo aleatorio simple. Del examen, resultaron significativos (pues en conjunto explicaban en más de un $93 \%$ las preferencias del alumnado) 5 atributos y 11 niveles de estudio, siendo éstos los siguientes: nivel de exigencia (alto, bajo), tipo de clase (igual número de horas de teoría que de práctica, menor número de horas de teoría que de práctica y mayor número de horas de teoría que de práctica), uso de Tecnologías de la Información y Comunicación (TIC) en el aula (tradicional, multimedia), número de profesores (un único profesor para la teoría y la práctica, más de un profesor para la teoría y la práctica) y el estilo docente (autocrático, integrador). La Tabla 1, detalla la definición de los atributos utilizados en el estudio.

Tabla 1: Atributos y niveles utilizados en el análisis de las preferencias

\begin{tabular}{|l|l|}
\hline Atributo & Definición \\
\hline $\begin{array}{l}\text { Nivel de } \\
\text { exigencia }\end{array}$ & Grado de exigencia de la clase, que puede ser alto o bajo. \\
\hline Tipo de clase & $\begin{array}{l}\text { Distribución de las horas teóricas y prácticas de la clase, que pueden ser iguales, mayormente de } \\
\text { práctica o de teoría. }\end{array}$ \\
\hline Uso de TIC & $\begin{array}{l}\text { Tipo de tecnología utilizada en clase. Puede ser: tradicional (pizarrón, libros y guías impresas) o } \\
\text { multimedia (publicaciones online, videos, e-books, Youtube, WhatsApp, etc.) }\end{array}$ \\
\hline $\begin{array}{l}\text { Número de } \\
\text { profesores }\end{array}$ & Cantidad de profesores que imparten la teoría y la práctica. Puede ser sólo uno o más de uno. \\
\hline $\begin{array}{l}\text { Estilo } \\
\text { docente }\end{array}$ & $\begin{array}{l}\text { Arte y técnica de enseñanza empleada por el docente. Puede ser autocrático (dominante, autoritario) o } \\
\text { integrador (participativo, democrático). }\end{array}$ \\
\hline
\end{tabular}

Para la recogida de datos, se aplicaron encuestas personales a una muestra total de 58 estudiantes de primer año de la carrera de Ingeniería Comercial, a través de un muestreo aleatorio simple, con una tasa de respuesta del $100 \%$. El tamaño muestral consideró un nivel de confianza del $95 \%$, un margen de error del $4,5 \%$ y fue obtenido sobre una población de 65 alumnos. Mediante el diseño ortogonal de los datos se definieron 8 perfiles de evaluación (tarjetas), que se les solicitó ordenar a los encuestados de la más a la menos preferida utilizando el procedimiento de perfil total con presentación verbal, el que se presenta en la Tabla 2.

Tabla 2: Perfiles de las tarjetas generadas para la investigación

\begin{tabular}{|c|c|l|l|l|c|}
\hline ID & Nivel de Exigencia & \multicolumn{1}{|c|}{ Tipo de Clase } & Uso de TIC & \multicolumn{1}{|c|}{ Número de Profesores } & Estilo Docente \\
\hline 1 & Alto & $\begin{array}{l}\text { Igual número de horas de } \\
\text { teoría que de práctica }\end{array}$ & Tradicional & $\begin{array}{l}\text { Un único profesor para la } \\
\text { teoría y la práctica }\end{array}$ & Autocrático \\
\hline 2 & Bajo & $\begin{array}{l}\text { Igual número de horas de } \\
\text { teoría que de práctica }\end{array}$ & Multimedia & $\begin{array}{l}\text { Más de un profesor para } \\
\text { la teoría y la práctica }\end{array}$ & Integrador \\
\hline 3 & Bajo & $\begin{array}{l}\text { Igual número de horas de } \\
\text { teoría que de práctica }\end{array}$ & Multimedia & $\begin{array}{l}\text { Un único profesor para la } \\
\text { teoría y la práctica }\end{array}$ & Autocrático \\
\hline 4 & Alto & $\begin{array}{l}\text { Mayor número de horas de } \\
\text { teoría que de práctica }\end{array}$ & Multimedia & $\begin{array}{l}\text { Más de un profesor para } \\
\text { la teoría y la práctica }\end{array}$ & Autocrático \\
\hline 5 & Alto & $\begin{array}{l}\text { Menor número de horas de } \\
\text { teoría que de práctica }\end{array}$ & Multimedia & $\begin{array}{l}\text { Un único profesor para la } \\
\text { teoría y la práctica }\end{array}$ & Integrador \\
\hline 6 & Bajo & $\begin{array}{l}\text { Menor número de horas de } \\
\text { teoría que de práctica }\end{array}$ & Tradicional & $\begin{array}{l}\text { Más de un profesor para } \\
\text { la teoría y la práctica }\end{array}$ & Autocrático \\
\hline 7 & Alto & $\begin{array}{l}\text { Igual número de horas de } \\
\text { teoría que de práctica }\end{array}$ & Tradicional & $\begin{array}{l}\text { Más de un profesor para } \\
\text { la teoría y la práctica }\end{array}$ & Integrador \\
\hline 8 & Bajo & $\begin{array}{l}\text { Mayor número de horas de } \\
\text { teoría que de práctica }\end{array}$ & Tradicional & $\begin{array}{l}\text { Un único profesor para la } \\
\text { teoría y la práctica }\end{array}$ & Integrador \\
\hline
\end{tabular}


El modelo conceptual utilizado como base para la regresión fue el modelo aditivo siguiente:

$U_{t}=\alpha+\beta_{1} x_{1 i}+\beta_{2} x_{2 j}+\beta_{3} x_{3 k}+\beta_{4} x_{4 l}+\beta_{5} x_{5 m}+\epsilon_{t}$

En la ecuación, $U_{t}$ representa la preferencia establecida por el t-ésimo individuo consultado, $x_{1 i}$ es la variable nivel de exigencia $\left(x_{11}=\right.$ alto y $x_{12}=$ bajo), $x_{2 j}$ estilo de la clase $\left(x_{21}=\right.$ igual número de horas de teoría que de práctica, $x_{22}=$ menor número de horas de teoría que de práctica y $x_{23}=$ mayor número de horas de teoría que de práctica), $x_{3 \mathrm{k}}$ uso de TIC en el aula ( $x_{31}=$ tradicional y $x_{32}=$ multimedia), $x_{41}$ número de profesores ( $x_{41}=$ un único profesor para la teoría y la práctica y $x_{42}=$ más de un profesor para la teoría y la práctica) y $x_{5 \mathrm{~m}}$ estilo docente $\left(x_{51}=\right.$ autocrático y $x_{52}=$ integrador), $\alpha$ es la constante de la regresión; $\beta_{1}, \beta_{2}, \beta_{3}, \beta_{4}$ y $\beta_{5}$ son los valores de utilidad parciales asociados a los niveles de cada atributo, y $\varepsilon_{t}$ es el error de estimación. Para medir el grado de ajuste del modelo se utilizó el Tau-b de Kendall. Por último, tanto los resultados del análisis de las preferencias (análisis conjunto) como el examen de las preguntas de caracterización de los alumnos, fueron realizados mediante el software PASW Statistics 18.0 para Windows.

\section{RESULTADOS Y DISCUSIÓN}

La Tabla 3 recoge los resultados obtenidos de la composición socio-demográfica de la muestra de estudio. En cuanto a la evaluación de la utilidad individual y agregada de los distintos niveles de cada atributo, la Tabla 4 muestra que la clase preferida por los estudiantes es aquella que posee un alto nivel de exigencia $(0,457)$, con igual número de horas de teoría y de práctica $(0,517)$, que utiliza tecnología multimedia $(0,267)$, con un único profesor para la teoría y la práctica $(0,272)$ y que emplea un estilo de enseñanza docente integrador $(0,556)$. La utilidad total (valoración) de este perfil de clase es de 6,44. Cabe destacar, que no se observaron mayores diferencias al comparar las preferencias por género o por edad. Con todo, se aprecia una inclinación de los alumnos de mayor edad por clases centradas más en lo práctico que en lo teórico, con una leve propensión por estilos de enseñanza de tipo colaborativo.

Tabla 3: Composición de la muestra de estudio

\begin{tabular}{|c|c|c|c|c|c|}
\hline Variable & Característica & Porcentaje & Variable & Característica & Porcentaje \\
\hline \multirow{2}{*}{ Sexo } & Masculino & 50 & \multirow{3}{*}{ Edad } & Menos de 19 años & 24 \\
\hline & Femenino & 50 & & Entre 19 y 23 años & 66 \\
\hline \multirow{3}{*}{ Residencia } & La Serena & 64 & & Más de 23 años & 10 \\
\hline & Coquimbo & 26 & \multirow{3}{*}{$\begin{array}{l}\text { Establecimiento } \\
\text { educacional de origen }\end{array}$} & Municipal & 22 \\
\hline & Otra & 10 & & Particular Subvencionado & 73 \\
\hline \multirow{2}{*}{ Ocupación } & Trabaja y estudia & 29 & & Particular & 5 \\
\hline & Estudia & 71 & Total de encuestados & 58 estudiantes & 100 \\
\hline
\end{tabular}

Por su parte, para determinar la contribución de cada uno de los atributos en las preferencias indicadas por los alumnos, se calcularon los puntajes de importancia relativa totales, por género y edad, véase la Tabla 5. En ella se observa que para el total de los alumnos el atributo más importante es el Tipo de clase, seguidos por el Estilo docente y el Nivel de exigencia, y concluye con el Número de profesores y el Uso de TIC. Lo anterior, pone de manifiesto que la articulación equilibrada entre la teórica y la práctica, es el factor más valorado en la dictación de una clase, muy por sobre otros, como el estilo docente o nivel de exigencia. Este hallazgo, si bien coincide con lo expuesto por Ramírez et al. (2004), es interesante, porque su importancia relativa se mantiene, aun cuando se mide en relación a otros atributos y otra asignatura (matemáticas).

Los resultados previos, además, permiten confirmar las preferencias expuestas por: Asún et al. (2013) y Huaquín y Loaíza (2004) respecto del nivel de exigencia alto; Boyer et al. (2009), Robinson y Stubberud (2012) y Tárraga et al. (2012) sobre la importancia de la utilización de herramientas multimedia en la labor docente. Asimismo, corroboran, lo señalado por Ramírez et al. (2004) sobre el número de profesores. Igualmente, el estilo pedagógico integrador del docente mostró ser concurrente a lo revelado por Cerdá y Ramírez (2010), Cui (2013), González-Peiteado (2013), Maureira y Rojas (2013) y Rojas-Jara et al. (2016). 
Tabla 4: Utilidades estimadas por el modelo conjunto

\begin{tabular}{|c|c|c|c|c|c|c|c|}
\hline Atributo & Nivel & Alumnos & Hombres & Mujeres & < 19 años & 19-23 años & > 23 años \\
\hline \multirow{2}{*}{$\begin{array}{l}\text { Nivel de } \\
\text { Exigencia }\end{array}$} & Alto & 0,457 & 0,491 & 0,422 & 0,286 & 0,533 & 0,375 \\
\hline & Bajo & $-0,457$ & $-0,491$ & $-0,422$ & $-0,286$ & $-0,533$ & $-0,375$ \\
\hline \multirow{3}{*}{$\begin{array}{l}\text { Tipo de } \\
\text { Clase }\end{array}$} & Teoría = Práctica & 0,517 & 0,402 & 0,632 & 0,786 & 0,465 & 0,222 \\
\hline & Teoría < Práctica & 0,474 & 0,376 & 0,572 & 0,411 & 0,379 & 1,222 \\
\hline & Teoría > Práctica & $-0,991$ & $-0,779$ & $-1,204$ & $-1,196$ & $-0,844$ & $-1,444$ \\
\hline \multirow{2}{*}{ Uso de TIC } & Tradicional & $-0,267$ & $-0,310$ & $-0,224$ & $-0,482$ & $-0,197$ & $-0,208$ \\
\hline & Multimedia & 0,267 & 0,310 & 0,224 & 0,482 & 0,197 & 0,208 \\
\hline \multirow{2}{*}{$\begin{array}{l}\mathrm{N}^{\circ} \text { de } \\
\text { profesores }\end{array}$} & Un único profesor & 0,272 & 0,319 & 0,224 & 0,518 & 0,237 & $-0,083$ \\
\hline & Más de un profesor & $-0,272$ & $-0,319$ & $-0,224$ & $-0,518$ & $-0,237$ & 0,083 \\
\hline \multirow{2}{*}{$\begin{array}{l}\text { Estilo } \\
\text { Docente }\end{array}$} & Autocrático & $-0,556$ & $-0,517$ & $-0,595$ & $-0,714$ & $-0,507$ & $-0,500$ \\
\hline & Integrador & 0,556 & 0,517 & 0,595 & 0,714 & 0,507 & 0,500 \\
\hline & Constante & 4,371 & 4,399 & 4,342 & 4,304 & 4,384 & 4,444 \\
\hline
\end{tabular}

Tabla 5: Importancia relativa de los atributos (porcentaje)

\begin{tabular}{|l|c|c|c|c|c|c|}
\hline \multicolumn{1}{|c|}{ Atributo } & Alumnos & Hombres & Mujeres & $<19$ años & $19-23$ años & $>23$ años \\
\hline Nivel de Exigencia & 17,708 & 19,307 & 16,108 & 15,942 & 18,850 & 14,590 \\
\hline Tipo de Clase & 34,019 & 33,947 & 34,091 & 31,983 & 32,353 & 49,324 \\
\hline Uso de TIC & 12,450 & 12,233 & 12,667 & 12,120 & 13,150 & 8,787 \\
\hline$N^{\circ}$ de profesores & 15,648 & 16,499 & 14,797 & 16,507 & 15,653 & 13,610 \\
\hline Estilo Docente & 20,175 & 18,014 & 22,337 & 23,447 & 19,994 & 13,689 \\
\hline
\end{tabular}

En otro sentido, se aprecia que el tipo de clase sumado al estilo docente explican en más de un $50 \%$ las preferencias de los estudiantes $(54,2 \%)$ lo que, a nivel particular, representan una valiosa información para corroborar la correspondencia del estudiantado con el Modelo Educativo de la Universidad de La Serena, que considera al estudiante como centro de su quehacer académico, con un rol participativo y activo de su propia formación. Esto implica plantearse el desafío de la alineación constructivista de la enseñanza universitaria propuesta por Biggs (2014) dado que muchas universidades siguen respondiendo a un sistema educativo tradicional y conductista, centrado en el profesor con estilo autocrático y con clases expositivas.

Por otra parte, el contraste de los niveles de importancia relativa por género, demuestra que no existen diferencias para el atributo más valorado (tipo de clase) ni para los menos valorados (número de profesores y uso de TIC), sin embargo, se observa que las mujeres dan mayor énfasis al estilo docente (22,337), destacándolo en segundo lugar de sus preferencias, mientras que los hombres califican en dicha posición al nivel de exigencia $(19,307)$. Esta diferencia, encuentra coincidencia con algunos estudios que indican que las mujeres como grupo poseen una serie de valores distintivos (valoran la relación, la colaboración y la comunicación) que las llevan apreciar más un estilo orientado hacia las necesidades de las personas, la cooperación, democracia y el trabajo en equipo, mientras que los hombres son más individualistas y prefieren un estilo más directivo (Eagly, 2013 y Shambaugh, 2016) vinculado a la competitividad y recompensas.

Situación similar se presenta al comparar los resultados obtenidos por edades, pues el tipo de clase fue el atributo más valorado y el uso de TIC el menos valorado. Sin embargo, lo llamativo, más allá de confirmar que los más jóvenes prefieren más la tecnología multimedia que los adultos (rasgo generacional), fue el descubrir el bajo peso relativo (menor al 9\%) que le otorgan los estudiantes de mayor edad (mayores de 23 años) al uso de este tipo de método (casi un 30\% menos que la muestra general de alumnos), además de observar como, según el orden de preferencias (importancia relativa) de los atributos, el nivel de exigencia, 
va obteniendo mayor preponderancia en los estudiantes de mayor rango etario (4to lugar para los menores de 19 años, 3er lugar para los que tienen entre 19 y 23 años y 2 do lugar para los mayores de 23 años).

Desde una perspectiva práctica los resultados muestran la relevancia de: (i) atender al cumplimiento del programa de clases y los estándares de exigencia, mediando un criterio docente, que considere el límite de horas de estudio autónomo que posee el estudiante; (ii) incorporar/formar docentes con habilidades para impartir clases tanto teóricas como prácticas; (iii) asentar el uso de las tecnologías de la información como un medio social valorado para el proceso de aprendizaje y desempeño profesional; (iv) limitar la coenseñanza a casos específicos donde la capacidad de los docentes sea complementaria; (v) desarrollar un estilo docente centrado en el alumno, que establezca el autoaprendizaje como un pilar fundamental de su formación académica.

Por último, el coeficiente de Tau-b de Kendall y el coeficiente $R$ de Pearson reportaron valores cercanos a 1 indicando el alto grado de ajuste y la validez interna de las utilidades obtenidas. Asimismo, ambos son significativos con valor de probabilidades de $p$ cercanos a cero (significancia del $5 \%$ ), ratificando que existe una fuerte correlación entre las preferencias observadas y las estimadas por el modelo conjunto.

\section{CONCLUSIONES}

Las principales conclusiones obtenidas del análisis de los atributos preferidos de los estudiantes en la enseñanza de la asignatura Administración de Empresas de la carrera de Ingeniería Comercial son: 1) la clase preferida por los estudiantes, es aquella que posee un alto nivel de exigencia, con una distribución equilibrada entre las horas de teoría y práctica, que emplea tecnología multimedia, dictada por un solo profesor con un estilo de enseñanza docente integrador; 2 ) los atributos que explican mayormente (más del $70 \%$ ) las preferencias en la enseñanza de la asignatura, en orden de importancia son el tipo de clase, el estilo docente y el nivel de exigencia; 3 ) las mujeres aprecian más el estilo docente, mientras que los hombres el nivel de exigencia; 4) los estudiantes de mayor edad no ven el uso de las TIC como un atributo determinante de sus preferencias; y 5) se evidencia la fortaleza de la técnica de Análisis Conjunto para la medición de las preferencias del alumnado.

Finalmente, cabe mencionar que el tamaño muestral reducido del estudio, da pie a la realización de nuevas investigaciones centradas en lograr mayor representatividad en la medición de las preferencias de los estudiantes (nivel regional y/o nacional), junto con replicar la técnica de Análisis Conjunto en asignaturas consideradas críticas (altos niveles de repitencia) dentro de los planes de estudios de las carreras.

\section{REFERENCIAS}

Ainscow, M., Haciendo que las escuelas sean más inclusivas: lecciones a partir del análisis de la investigación internacional, Revista de Educación inclusiva, ISSN: 1889-4208 (en línea), 5(1), 39-49, 2012. https://goo.gl/pG4kqx. Acceso: 1 de Julio (2017)

Ajani, S., Impact of Information Communication Technology on Teaching and Learning of Business Education, Scholarly Journal of Education, ISSN: 2315-6155, (en línea), 5(1), 1-6, 2016. https://goo.gl/Xwqfm5. Acceso: 1 de Julio (2017)

Asun, R., Zúñiga, C. y Ayala, M., La formación por competencias y los estudiantes: confluencias y divergencias en la construcción del docente ideal, doi: 10.4067/S0718-45652013000100008, Revista Calidad en la educación, (en línea), (38), 277-304 (2013)

Biggs, J., Constructive alignment in university teaching, HERDSA Review of Higher Education, ISSN: 01571826 (en línea), 1(5), 5-22, 2014. https://goo.gl/CmFQiK, Acceso: 1 de Julio (2017)

Borgobello, A., Peralta, N. y Roselli, N., Estilo docente universitario en relación al tipo de clase y a la disciplina enseñada, Liberabit, ISSN: $1729-4827$ (en línea), 16(1), 7-16, 2010. https://goo.gl/faLP64. Acceso: 5 de Mayo (2016)

Boyer, T.A., Briggeman, B.C. y Norwood, F.B., Demand for multimedia in the classroom, doi: 10.1017/S1074070800003229, Journal of Agricultural and Applied Economics, (en línea), 41(3), 791-808 (2009)

Cerdá, L. y Ramírez, M., Evaluación de estilos de liderazgo en la docencia: una aplicación en la enseñanza universitaria de postgrado. Revista Iberoamericana de Sistemas, Cibernética e Informática, ISSN: 16908627 (en línea), 7(1), 55-62, 2010. https://goo.gl/jSGt2P. Acceso: 1 de Julio (2017) 
Cui, Y., An Empirical Study of Learning Outcomes Based on Active Versus Passive Teaching Styles, doi: 10.5815/ijeme.2013.01.07, I.J. Education and Management Engineering, (en línea), 1, 39-43 (2013)

Eagly, A. H., Women as leaders: Leadership style versus leaders' values and attitudes. In Gender and work: Challenging conventional wisdom, Harvard Business School Press (2013)

Farías, P. y Fistrovic, B., Las preferencias del consumidor usando el método de máximas diferencias, doi: 10.1590/S0034-759020160202, Revista de Administração de Empresas, (en línea), 56(2), 138-151 (2016)

García-Vidal, G., Pérez-Campdesuñer, R., Martínez-Vivar R. y Sánchez-Rodríguez, A., Conjoint analysis tool for determining attributes on designing a graduate degree program, doi: 10.18775/jibrm.18498558.2015.15.3005, Journal of International Business Research and Marketing, (en línea), 1(5), 33-38 (2016)

Gómez-Valenzuela, V., Analysis of Preference of Incentives to Innovation of Dominican Manufacturing and Service Firms, doi: 10.4067/S0718-27242015000200007, Journal of technology management \& innovation, (en línea), 10(2), 93-116 (2015)

González-Peiteado, M., Los estilos de enseñanza y aprendizaje como soporte de la actividad docente, Revista Estilos de Aprendizaje, ISSN: $2332-8533$ (en línea), 6(11), 51-65, 2013. https://goo.gl/iWCfAo. Acceso: 1 de Julio (2017)

Hernández-Infante, R.C. e Infante-Miranda, M.E., La clase en la educación superior, forma organizativa esencial en el proceso de enseñanza-aprendizaje, doi: 10.5294/edu.2017.20.1.2, Pedagogía Universitaria, (en línea), 20(1), 27-40 (2017)

Huaquín, V. y Loaíza, R., Exigencias académicas y estrés en las carreras de la Facultad de Medicina de la Universidad Austral de Chile, doi: 10.4067/S0718-07052004000100003, Estudios pedagógicos (Valdivia), (en línea), (30), 39-59 (2004)

Huertas-García, R., Nuñez-Carballosa, A. y Miravitlles, P., Statistical and cognitive optimization of experimental designs analysis, doi: 10.1016/j.redee.2015.10.001, Revista Europea de Dirección y Economía de la Empresa, (en línea), 25(3), 142-149 (2016)

Kharb, P., Samanta, P.P., Jindal, M. y Singh, V., The learning styles and the preferred teaching-learning strategies of first year medical students, doi: 10.7860/JCDR/2013/5809.3090, Journal of clinical and diagnostic research: JCDR, (en línea), 7(6), 1089-1092 (2013)

Kuzmanovic, M., Savic, G., Popovic, M. y Martic, M., A New Approach to Evaluation of University Teaching Considering Heterogeneity of Students' Preferences, doi: 10.1007/s10734-012-9596-2, Higher Education, (en línea), 66(2), 153-171 (2013)

López, S. y Paredes, L., Análisis exploratorio de los planes de estudio de Ingeniería Comercial en Chile, Pensamiento \& Gestión, ISSN: 1657-6276 (en línea), 23, 58-71, 2007. https://goo.gl/6gq1BE. Acceso: 1 de Julio (2017)

Louviere, J.J., Hensher, D.A. y Swait, J.D., Stated choice methods: analysis and applications, ${ }^{\text {a }}$ Ed., 34-62. Cambridge University Press, Cambridge, Inglaterra (2000)

Maureira, O. y Rojas, A., Características del Liderazgo Transformacional en Docentes de Lenguaje y Comunicación en una Muestra de Establecimientos Escolares de Vulnerabilidad Social, doi: 10.4067/S071807052013000100007, Estudios pedagógicos (Valdivia), (en línea), 39(1), 115-127 (2013)

Nicholson W. y Snyder, C., Microeconomic Theory: basic principles and extensions, 10 ${ }^{\mathrm{a}}$ Ed., 87-105. Thomson/South-Western, Ohio, Estados Unidos (2008)

Ramírez, J. M., Fedriani, E. y Melgar, M. del C., Determinación del perfil de docencia preferido por los alumnos de matemáticas de LADE, Rect@, ISSN: 1575-605X (en línea), 12(1), 16-26, 2004. https://goo.gl/iACaHh. Acceso: 1 de Abril (2016) 
Ramos, A., La colaboración docente en la educación superior. Revista de Educación de la Universidad de Granada, ISSN: 0214-0489, (en línea), 23, 267-279, 2016. https://goo.gl/Z7Xf5Q. Acceso: 1 de Julio (2017)

Robinson, S. y Stubberud, H. A., Student preferences for educational materials: old meets new, Academy of Educational Leadership Journal, ISSN: 1528-2643 (en línea), 16(S1), 99-109 (2012)

Rojas-Jara, C., Díaz-Larenas, C., Vergara-Morales, J., Alarcón-Hernández, P. y Ortiz-Navarrete, M., Estilos de enseñanza y estilos de aprendizaje en educación superior: Análisis de las preferencias de estudiantes de Pedagogía en Inglés en tres universidades chilenas, doi: 10.15359/ree.20-3.7, Revista Electrónica Educare, 20(3), 141-169 (2016)

Shambaugh, R., The Fine Line Between a Collaborative Employee and One Who Doesn't Get Enough Done. Harvard Business Review (2016)

Suárez-Díaz G., Co-enseñanza: concepciones y prácticas en profesores de una Facultad de Educación en Perú. Revista Electrónica de Investigación Educativa, ISSN: 1607-4041 (en línea), 18(1), 166-182, 2016. https://goo.gl/gw55uQ. Acceso: 1 de Julio (2017)

Tárraga, R., Martínez, P., Gómez del Amo, R., Pastor, G. y Fernández, I., Análisis de las preferencias instruccionales de estudiantes universitarios: El papel del material de apoyo a las clases. Revista Iberoamericana de Informática Educativa, ISSN: 1699-4574 (en línea), 16, 69-77, 2012. https://goo.gl/jc7kee. Acceso: 2 de Marzo (2016)

Ubulom, W.J., Kayii, N., y Dambo, B.I., Information and Communication Technology and Business Studies Students' Academic Performance in Upper Basic Education in Tai Local Government Rivers State, Nigeria, International Journal of Innovative Social \& Science Education Research, ISSN: 2360-8978 (en línea), 4(1), 1-7, 2016. https://goo.gl/PG5KvK. Acceso: 1 de Julio (2017) 
